\title{
Diversidade e estrutura genética de populações de Podocarpus lambertii Klotzsch ex Endl. na floresta ombrófila mista em Santa Catarina
}

\author{
Genetic diversity and structure of Podocarpus lambertii Klotzsch ex Endl. \\ populations in the mixed ombrophilous forest of Santa Catarina state
}

\author{
Ricardo Bittencourt ${ }^{\mathrm{I}}$, Felipe Steiner ${ }^{\mathrm{II}}$, Cristina Silva Sant'Anna ${ }^{\mathrm{III}}$, Tiago Montagna ${ }^{\mathrm{IV}}$, \\ Caio Darós Fernandes ${ }^{\mathrm{V}}$, Fernando André Loch Santos da Silvav ${ }^{\mathrm{V}}$, Maurício Sedrez dos Reis ${ }^{\mathrm{VI}}$
}

\begin{abstract}
Resumo
Podocarpus lambertii (Podocarpaceae) é conhecido popularmente como pinheiro-bravo. Ocorre em várias formações florestais da Mata Atlântica, principalmente na Floresta Ombrófila Mista. Devido à intensa exploração madeireira sofrida pela espécie, ela está presente na categoria "Quase ameaçada" da Lista Vermelha das Espécies Ameaçadas da IUCN. Diante disso, este estudo teve por objetivo caracterizar a diversidade e estrutura genética de 12 populações no Estado de Santa Catarina, a fim de gerar informações para fundamentar estratégias de conservação para a espécie. Foram coletadas folhas de 50 indivíduos adultos em cada população. Para acessar os níveis e a distribuição da diversidade genética, utilizaram-se 10 sistemas enzimáticos. As frequências alélicas, a porcentagem de locos polimórficos $\left(P_{100}\right)$, as heterozigosidades observada $\left(H_{\mathrm{O}}\right)$ e esperada $\left(H_{\mathrm{E}}\right)$, o índice de fixação $(f)$ e a divergência genética $\left(F_{S T}\right)$ foram calculados com auxílio do programa FSTAT. Os 10 sistemas enzimáticos analisados revelaram 11 locos com um total de 32 alelos $(\mathrm{A}=1,8)$. As populações de Podocarpus lambertii apresentaram baixa diversidade genética $\left(P_{100 \%}=52,7 ; H_{0}=0,049\right.$; $\left.H_{\mathrm{E}}=0,079\right)$. As frequências alélicas das populações apresentaram desvios significativos das esperadas em Equilíbrio de Hardy-Weinberg, evidenciando um deficit de heterozigotos $(f=0,374)$. A presença de alelos raros nas populações e uma divergência genética significativa $\left(F_{\mathrm{ST}}=0,303\right)$ evidenciam um baixo fluxo gênico histórico e um grande risco de perda de diversidade. Os resultados obtidos indicam a necessidade de conservação in situ das várias populações de Podocarpus lambertii e de um aumento da conectividade entre as populações remanescentes.
\end{abstract}

Palavras-chave: Alozimas; Índice de fixação; Conservação in situ; Conífera

\begin{abstract}
Podocarpus lambertii (Podocarpaceae) is popularly known in Brazil as pinheiro-bravo. It occurs in several forest formations of the Atlantic Rain Forest, mainly in the Ombrophilous Mixed Forest. Due to the high wood exploitation suffered by the species, it is listed as 'Almost Threatened' in The IUCN Red List of Threatened Species. In face of this, the objective of the present study was to characterize the genetic diversity and structure of 12 natural populations of Podocarpus lambertii in Santa Catarina state, aiming to generate information for the definition of effective conservation strategies for the species. To access genetic diversity levels and distribution, it was used 10 allozymic systems. Along with the assistance of the software Fstat, the allelic frequencies (A), polymorphic loci percentage (P), observed heterozigosity $\left(\mathrm{H}_{\mathrm{o}}\right)$, expected heterozigosity, fixation index $(f)$ and genetic divergence between populations $\left(F_{S T}\right)$ were calculated. In 11 loci 32 alleles $(\mathrm{A}=1,8)$ were detected. A low genetic diversity was detected for Podocarpus lambertii populations $\left(P_{100 \%}=52,7 ; H_{\mathrm{O}}=0,049 ; H_{\mathrm{E}}=0,079\right)$. The mean fixation index obtained $(f=0,374)$ indicates a deficit of heterozygous individuals. A high number of low frequency alleles was detected and together with the high genetic divergence $\left(F_{\mathrm{ST}}=0,303\right)$, it points towards the necessity of in situ conservation of a large number of Podocarpus lambertii populations and the need to increase the connectivity among the remnant populations.
\end{abstract}

Keywords: Allozymes; Fixation index; In situ conservation; Conifer

\footnotetext{
I Engenheiro Agrônomo, Dr., Professor do Departamento de Ciências Naturais, Centro de Ciências Exatas e Naturais, Universidade Regional de Blumenau, Rua Antônio da Veiga, 140, CEP 89030-903, Blumenau (SC), Brasil. bittencourtr@gmail.com (ORCID: 0000-0002-6609-3372)

II Engenheiro Florestal, MSc., Pesquisador Autônomo, Núcleo de Pesquisas em Florestas Tropicais, Departamento de Fitotecnica, Centro de Ciências Agrárias, Universidade Federal de Santa Catarina, Rod. Admar Gonzaga, 1346, CEP 88.034-001, Florianópolis (SC), Brasil. lipesteiner21@gmail.com (ORCID: 0000-0002-0200-8589)

III Bióloga, MSc., Professora do Departamento de Ciências Naturais, Centro de Ciências Exatas e Naturais, Universidade Regional de Blumenau, Rua Antônio da Veiga, 140, CEP 89030-903, Blumenau (SC), Brasil. cssanna@furb.br (ORCID: 0000-0002-1286-5250)

${ }^{I V}$ Engenheiro Agrônomo, MSc., Doutorando do Programa de Pós-Graduação em Recursos Genéticos Vegetais, Centro de Ciências Agrárias, Universidade Federal de Santa Catarina, Rod. Admar Gonzaga, 1346, CEP 88.034-001, Florianópolis (SC), Brasil. monttagna@gmail.com (ORCID:0000-0002-7427-4237)

v Engenheiro Agrônomo, Pesquisador Autônomo, Núcleo de Pesquisas em Florestas Tropicais, Departamento de Fitotecnica, Centro de Ciências Agrárias, Universidade Federal de Santa Catarina, Rod. Admar Gonzaga, 1346, CEP 88.034-001, Florianópolis (SC), Brasil. caiodaros@gmail.com (ORCID: 0000-0002-5608-3277)/ andloch@gmail.com (ORCID: 0000-0001-8722-4063)

VI Engenheiro Agrônomo, Dr., Professor do Departamento de Fitotecnia, Centro de Ciências Agrárias, Universidade Federal de Santa Catarina, Rod. Admar Gonzaga, 1346, CEP 88.034-001, Florianópolis (SC), Brasil. msedrez@gmail.com (ORCID: 0000-0002-1486-0242)
} 


\section{Introdução}

A Mata Atlântica é um dos ecossistemas mais devastado e ameaçado do planeta, sendo reconhecido como um dos 25 hotspots de biodiversidade do mundo (MYERS et al., 2000). O Estado de Santa Catarina era, originalmente, totalmente coberto pela Mata Atlântica, sendo que dentro deste bioma, a área de domínio da Floresta Ombrófila Mista engloba 42,5\% do território estadual. No estado, as estimativas apontam que existam cerca de 22,4\% de remanescentes de Mata Atlântica, o estado com o maior percentual de remanescentes. Porém, Santa Catarina também é apontado como o estado com o maior índice de desflorestamento, cerca de $1,19 \%$ dos remanescentes foram perdidos de 2008 até 2010 (FUNDAÇÃO SOS MATA ATLÂNTICA; INSTITUTO NACIONAL DE PESQUISAS ESPACIAIS, 2010).

A substituição da floresta por atividades antrópicas, levando à redução do tamanho das populações, causa um gargalo genético devido ao fato de que os indivíduos remanescentes contêm apenas uma pequena amostra do pool gênico original. Com as populações permanecendo pequenas e isoladas umas das outras, a deriva genética passa a ter uma influência dominante na estrutura genética das mesmas, podendo levar à perda de variabilidade (BARRET; KHON, 1991; YOUNG; BROWN, 1999).

Considerando-se os processos que resultam da fragmentação, as populações presentes nos fragmentos podem apresentar uma redução do potencial evolutivo aumentado a sua probabilidade de extinção local devido a aspectos como aumento da depressão endogâmica, perda de diversidade genética e maiores probabilidades de fixação de alelos deletérios, quando comparados com a estrutura populacional pré-fragmentação (DUDASH; FENSTER, 2000; FRANKHAM, 2005).

Para plantas, os efeitos genéticos da fragmentação de habitat geralmente são complicados pelo hábito séssil, diferenças de longevidade entre espécies, quantidade de gerações pós-fragmentação, abundância pré-fragmentação, grande variedade de sistemas reprodutivos sexuais e assexuais, a possibilidade de fluxo gênico por pólen e por semente, a possibilidade de conservar material genético como sementes e as interações com animais polinizadores e vetores de dispersão, que por si só são afetados pela fragmentação (YOUNG; BOYLE; BROWN, 1996).

Duas consequências genéticas do reduzido tamanho populacional são o aumento dos efeitos da endogamia e da deriva genética. A deriva genética é a mudança aleatória nas frequências alélicas que ocorre quando os gametas transmitidos de uma geração para a próxima levam somente uma parte dos alelos presentes na geração parental. A deriva genética muda a distribuição da variabilidade genética de duas formas: a perda de variação de populações e o aumento da divergência genética entre populações. Toda população finita sofre efeitos de deriva genética, mas os efeitos se tornam mais pronunciados conforme o tamanho populacional diminui (ELAM; ELLSTRAND, 1993). Quando populações se tornam pequenas, a deriva genética pode levar a uma redução na variabilidade genética que pode ser medida de diferentes modos. Conforme alelos são perdidos locos se tornam monomórficos e diferentes frequências alélicas nos locos polimórficos remanescentes devem reduzir a heterozigosidade esperada. O isolamento dos indivíduos remanescentes, também pode levar a uma redução nos níveis de heterozigosidade observada e a aumentos na taxa de endogamia (COLE, 2003). De acordo com Young, Boyle e Brown (1996), a perda de variabilidade está normalmente ligada mais fortemente à formação de gargalos genéticos no momento da fragmentação e à subsequente endogamia nas populações pequenas, do que aos efeitos de contínua deriva genética. Essa perda de diversidade genética em populações pequenas é responsável pelo aumento no risco de extinção por afetar adversamente a capacidade das populações de evoluir frente às mudanças ambientais (potencial evolutivo) (FRANKHAM, 2005).

A endogamia normalmente ocorre quando populações são pequenas ou quando elas apresentam estrutura genética espacial. A estrutura genética normalmente ocorre quando a dispersão de genes via pólen ou sementes é espacialmente restrita (ELAM; ELLSTRAND, 1993). A fragmentação reduz o número de indivíduos reprodutivos em uma população e, consequentemente, os cruzamentos em populações fragmentadas normalmente se dão por autofecundação (se possível) e/ou por cruzamentos entre aparentados, resultando em uma progênie endogâmica. A continuidade de cruzamentos endogâmicos, associados ao pequeno tamanho populacional devem resultar na perda de diversidade genética intrapopulacional, aumentando a homozigose nas populações (ELAM; ELLSTRAND, 1993; DUDASH; FENSTER, 2000). Em populações que sofrerão endogamia contínua, a frequência dos heterozigotos tende a se aproximar de zero (ELAM; ELLSTRAND, 1993). 
O entendimento do modo como a variabilidade genética está distribuída entre as populações é de importância primária para a conservação da diversidade genética, e consequentemente do potencial evolutivo, das espécies (HAMRICK; GODT, 1989). Uma das principais metas nos programas de conservação, adicionalmente a preservação de habitat, é a manutenção dos níveis existentes de diversidade genética em espécies raras, ameaçadas ou que estão inseridas em biomas ameaçados. A perda da variabilidade deve reduzir a habilidade das populações de se adaptarem às mudanças ambientais e aumentar a sua susceptibilidade a pressões de pestes e doenças (BARRET; KHON, 1991). Os estudos baseados em marcadores alozímicos podem ser utilizados para fornecer dados cruciais para o estabelecimento de estratégias que visam à preservação da diversidade genética (HAMRICK; GODT, 1989).

Dado o enorme impacto que a fragmentação pode ter na estrutura genética e nas dinâmicas ecológicas e evolutivas e, desse modo, na persistência das populações, é de fundamental importância que se faça uma integração das informações genéticas e de autoecologia na tentativa de definir estratégias efetivas de conservação, a fim de garantir a sobrevivência de espécies e populações a longo prazo (CLARKE; YOUNG, 2000; THRALL; BURDON; MURRAY, 2000).

Diante disso, o Governo do Estado de Santa Catarina incluiu nas Metas do Projeto Inventário Florístico Florestal de Santa Catarina (IFF-SC) a avaliação da diversidade genética de espécies da flora nativa ameaçadas de extinção, visando dar uma maior fundamentação às estratégias de conservação que serão delineadas após a conclusão do Projeto. Assim, o presente trabalho tem por objetivo apresentar os dados de diversidade e estrutura genética obtidos para uma espécie da Floresta Ombrófila Mista do Estado de Santa Catarina, Podocarpus lambertii Klotzsch ex Endl.

\section{Metodologia}

O Podocarpus lambertii, também conhecido como pinho-bravo ou pinheiro-bravo, é uma árvore perenifólia de altura variável, medindo de 1 a $4 \mathrm{~m}$ de altura na zona campestre e alcançando até $27 \mathrm{~m}$ de altura e $120 \mathrm{~cm}$ ou mais de DAP, na idade adulta, na Floresta com Araucária, onde comumente ocorre com $10 \mathrm{~m}$ de altura e 20 a $40 \mathrm{~cm}$ de DAP.

Apresenta tronco geralmente tortuoso, inclinado e curto, podendo apresentar-se reto na floresta, na qual atinge fustes de até $10 \mathrm{~m}$ de comprimento (CARVALHO, 1994). A ocorrência da espécie vai desde o Estado do Rio Grande do Sul até a Bahia, sendo encontrada também no nordeste da Argentina principalmente na área dominada pela Floresta Ombrófila Mista (RIBEIRO et al., 2007). Porém, a espécie é também encontrada na Floresta Estacional Decidual, na Floresta Estacional Decidual Montana, e nos Campos (CARVALHO, 1994).

Segundo Longhi (1995), o Podocarpus lambertii é uma espécie dioica, polinizada principalmente por abelhas e pequenos insetos. Longhi et al. (2010) afirmam que o Podocarpus lambertii prefere locais pouco pedregosos, pouco inclinados, relativamente úmidos, com alta frequência de indivíduos e alta densidade do sub-bosque, indicando ser esta uma espécie secundária tardia tolerante à sombra. De acordo com Carvalho (1994), a espécie é encontrada com excelente regeneração natural em capoeirões e vegetação secundária mais evoluída ou mesmo em capões.

Coleta

Foram coletadas amostras foliares em 12 populações da espécie na área de domínio da Floresta Ombrófila Mista no Estado de Santa Catarina. Estas populações estão distribuídas em quatro microrregiões do estado, sendo que em cada microrregião foram coletadas três populações. Alocalização das 12 populações naturais de Podocarpus lambertii coletadas no Estado de Santa Catarina para o desenvolvimento do presente trabalho podem ser visualizadas na Figura 1. 
Figura 1 - Locais de coleta de Podocarpus lambertii para a caracterização da diversidade e estrutura genética no Estado de Santa Catarina. UFSC, 2011.

Figure 1 - Assessment areas of Podocarpus lambertii for the characterization of genetic diversity and structure in Santa Catarina state. UFSC, 2011.

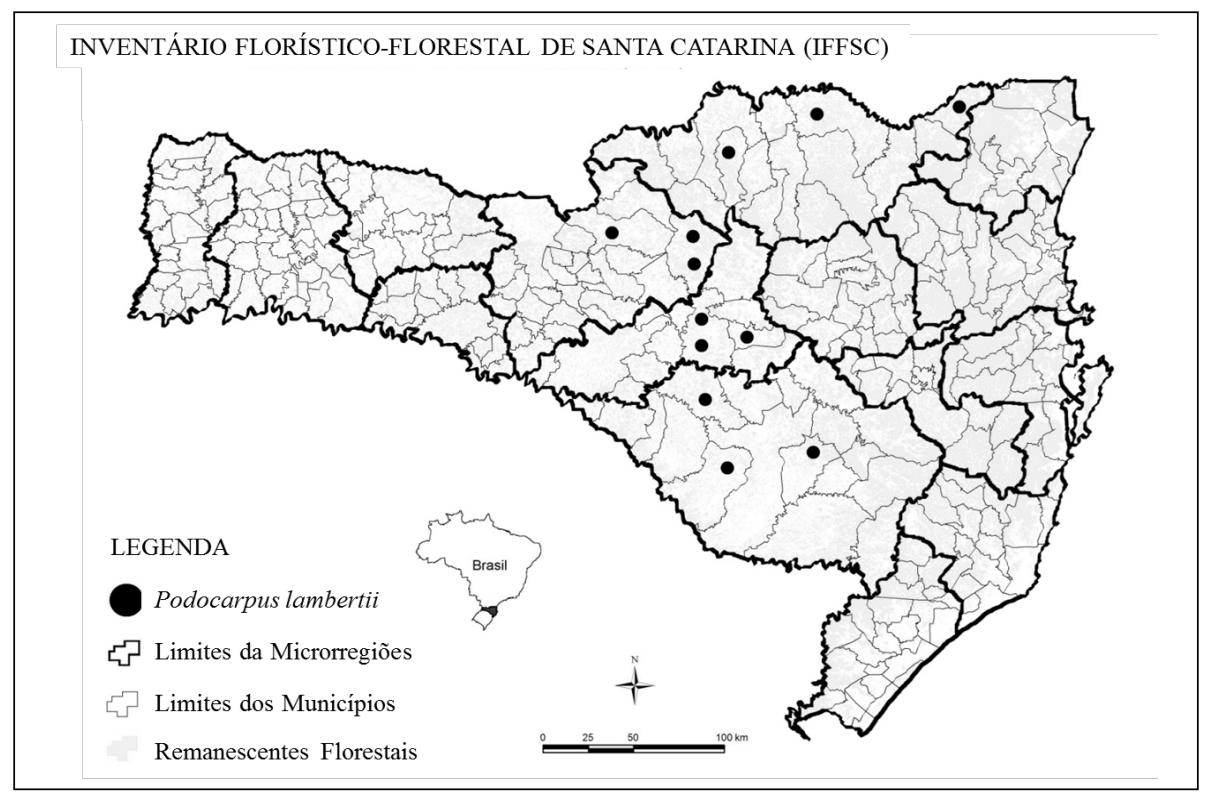

Acoleta das plantas foi realizada aleatoriamente, abrangendo apenas os indivíduos adultos distribuídos nas áreas de coleta, procurando, na medida do possível, respeitar uma distância mínima de 50 metros entre os indivíduos selecionados. Buscou-se coletar no mínimo 50 indivíduos por população, visto que esse é um número considerado satisfatório por Berg e Hamrick (1997) para o marcador empregado. A amostragem foi feita coletando-se pelo menos um ramo portador de folhas maduras dos indivíduos selecionados.

Para cada população coletada também foram levantadas informações do ambiente florestal em que estavam inseridas (Tabela 1).

Tabela 1-Número da Unidade Amostral de referência do IFF-SC, nome da população e características do fragmento onde foi realizada a coleta de 12 populações de Podocarpus lambertii. UFSC, 2011.

Table 1 - Number of the reference sample unit of IFF-SC, name of the population and characteristics of the fragment where the collection of 12 populations of Podocarpus lambertii was carried out. UFSC, 2011.

\begin{tabular}{c|c|c}
\hline UA & Município & Características \\
\hline 453 & S. Cristovão & Grande remanescente florestal em estágio médio de regeneração em que ocorreu exploração \\
madeireira & $\begin{array}{c}\text { Grande remanescente florestal em estágio médio de regeneração em que ocorreu exploração } \\
\text { madeireira }\end{array}$ \\
211 & Painel & Pequeno remanescente florestal em estágio médio de regeneração em que ocorreu exploração \\
madeireira
\end{tabular}


Tabela 2 - Conclusão...

Table 2 - Conclusion...

\begin{tabular}{c|c|c}
\hline UA & Município & Características \\
\hline 409 & Curitibanos 2 & $\begin{array}{c}\text { Pequeno remanescente florestal em estágio médio de regeneração em que ocorreu exploração } \\
\text { madeireira }\end{array}$ \\
784 & Caçador & $\begin{array}{c}\text { Pequeno remanescente florestal em estágio médio de regeneração em que ocorreu exploração } \\
\text { madeireira }\end{array}$ \\
1063 & Campo Alegre & Grande remanescente florestal em bom estado de conservação \\
1042 & Mafra & Grande remanescente florestal em estágio médio de regeneração em que ocorreu exploração \\
madeireira
\end{tabular}

\section{Eletroforese de isoenzimas}

O meio suporte utilizado para o desenvolvimento do presente trabalho foi o gel de amido de milho (penetrose 30) - 13\%. A extração das enzimas foi realizada com aproximadamente $7 \mathrm{mg}$ de Polivinil Pirrolidona (PVP), 30 mg de areia lavada e duas gotas de solução de extração no 1 de Alfenas et al. (1998). O sistema tampão eletrodo-gel utilizado para as corridas eletroforéticas foi o sistema Tris-Citrato. Para a revelação das bandas foram utilizados os seguintes sistemas isoenzimáticos: ME, MDH, DIA, PGI, IDH, 6PGDH, PGM, GTDH, G2DH e G6PDH.

Análise dos dados

A partir dos genótipos obtidos para cada indivíduo, foram calculadas, para cada população, as frequências alélicas e os índices de diversidade intrapopulacional, sendo eles a porcentagem de locos polimórficos $(P)$, número de alelos por loco $(A)$, heterozigosidade observada $\left(H_{o}\right)$ e heterozigosidade esperada $\left(H_{e}\right)$, além do índice de fixação $(f)$. Também foram calculados os estimadores de estruturação populacional de Weir e Cockerham (1984) e o índice $G_{S T}$ 'de Hedrick (2005). Tanto os índices de diversidade intrapopulacional, quanto os estimadores de estruturação foram calculados também para as populações dentro de microrregiões e bacias hidrográficas nos quais foram realizadas as coletas. Para a obtenção destes índices foram utilizados os programas FSTAT versão 2.9.3.2 (GOUDET, 2002) e GDA (LEWIS; ZAYKIN, 2001).

Buscando verificar algum padrão de agrupamento entre as populações estudadas, foram gerados, com o auxílio do programa FSTAT versão 2.9.3.2 (GOUDET, 2002), dendrogramas através do método UPGMA a partir das distâncias genéticas de Nei (1972) obtidas entre as populações e a estabilidade dos nós foi verificada pelo método de reamostragem bootstrap (10.000 repetições). Do mesmo modo, foram calculadas para todas as populações, as divergências genéticas $\left(F_{S T}\right)$ par a par. Com base nestes dados e nas distâncias geográficas das populações foi realizado um teste de Mantel para verificar se as distâncias geográficas entre as populações possuíam correlação com as divergências genéticas entre as mesmas. $\mathrm{O}$ Teste de Mantel foi realizado utilizando o logaritmo natural da distância geográfica e $\mathrm{F}_{\mathrm{ST}} /\left(1-\mathrm{F}_{\mathrm{ST}}\right)$. A significância do teste foi determinada através de 1000 permutações com o auxílio do programa NTSYS (ROHLF, 1989).

\section{Resultados e discussão}

Para acessar os níveis e a distribuição da diversidade genética, foram empregados dez sistemas enzimáticos, que revelaram os 11 locos utilizados para a caracterização genética das populações da espécie. Foram detectados 32 alelos para as 12 populações de Podocarpus lambertii e, na Tabela 2, podem ser visualizadas como estão distribuídas as frequências destes alelos nas diferentes populações. 
Bittencourt, R.; Steiner, F.; Sant'Anna, C. S.; Montagna, T.; Fernandes, C. D.;

Tabela 2 - Frequências alélicas para 12 populações de Podocarpus lambertii avaliadas no Estado de Santa Catarina. UFSC, 2011.

Table 2 - Allelic frequencies from 12 populations of Podocarpus lambertii in Santa Catarina state. UFSC, 2011.

\begin{tabular}{|c|c|c|c|c|c|c|c|c|c|c|c|c|c|c|}
\hline 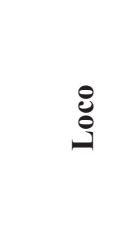 & $\frac{0}{\frac{0}{2}}$ & 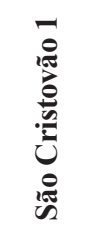 & 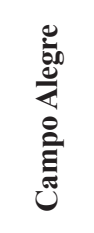 & $\frac{\pi}{\stackrel{\pi}{E}}$ & 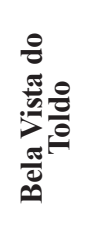 & 离 & ن & 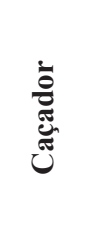 & 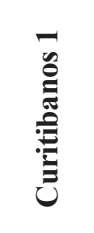 & 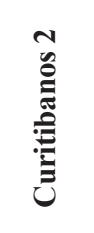 & 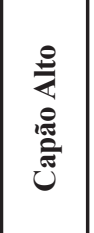 & 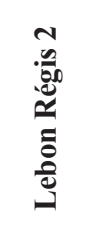 & 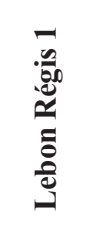 & है \\
\hline \multirow[t]{5}{*}{ MDH1 } & 1 & 0,057 & 0,221 & 0,173 & 0,034 & 0,000 & 0,000 & 0,000 & 0,000 & 0,000 & 0,027 & 0,000 & 0,000 & 0,043 \\
\hline & 2 & 0,000 & 0,029 & 0,000 & 0,000 & 0,000 & 0,000 & 0,000 & 0,000 & 0,000 & 0,000 & 0,000 & 0,000 & 0,002 \\
\hline & 3 & 0,028 & 0,087 & 0,018 & 0,009 & 0,045 & 0,018 & 0,008 & 0,000 & 0,059 & 0,045 & 0,089 & 0,000 & 0,034 \\
\hline & 4 & 0,915 & 0,663 & 0,809 & 0,957 & 0,955 & 0,964 & 0,992 & 1,000 & 0,941 & 0,909 & 0,911 & 1,000 & 0,918 \\
\hline & 5 & 0,000 & 0,000 & 0,000 & 0,000 & 0,000 & 0,018 & 0,000 & 0,000 & 0,000 & 0,018 & 0,000 & 0,000 & 0,003 \\
\hline ME1 & 1 & 1,000 & 1,000 & 1,000 & 1,000 & 1,000 & 1,000 & 1,000 & 1,000 & 1,000 & 1,000 & 1,000 & 1,000 & 1,000 \\
\hline \multirow[t]{3}{*}{ 6PGDH2 } & 1 & 0,000 & 0,000 & 0,000 & 0,000 & 0,000 & 0,000 & 0,000 & 0,007 & 0,000 & 0,018 & 0,089 & 0,000 & 0,010 \\
\hline & 2 & 0,972 & 0,925 & 0,982 & 0,845 & 1,000 & 0,926 & 1,000 & 0,979 & 0,980 & 0,964 & 0,875 & 1,000 & 0,954 \\
\hline & 3 & 0,028 & 0,075 & 0,018 & 0,155 & 0,000 & 0,074 & 0,000 & 0,014 & 0,020 & 0,018 & 0,036 & 0,000 & 0,037 \\
\hline \multirow[t]{4}{*}{ PGM1 } & 1 & 0,896 & 0,041 & 0,018 & 0,198 & 0,100 & 0,000 & 0,008 & 0,000 & 0,157 & 0,045 & 0,009 & 0,000 & 0,123 \\
\hline & 2 & 0,104 & 0,837 & 0,964 & 0,767 & 0,900 & 0,955 & 0,902 & 0,986 & 0,794 & 0,945 & 0,991 & 0,991 & 0,845 \\
\hline & 3 & 0,000 & 0,122 & 0,018 & 0,034 & 0,000 & 0,045 & 0,066 & 0,014 & 0,029 & 0,009 & 0,000 & 0,009 & 0,029 \\
\hline & 4 & 0,000 & 0,000 & 0,000 & 0,000 & 0,000 & 0,000 & 0,025 & 0,000 & 0,020 & 0,000 & 0,000 & 0,000 & 0,004 \\
\hline \multirow[t]{3}{*}{ PGM2 } & 1 & 0,019 & 0,077 & 0,100 & 0,017 & 0,176 & 0,045 & 0,016 & 0,029 & 0,049 & 0,027 & 0,000 & 0,000 & 0,046 \\
\hline & 2 & 0,943 & 0,894 & 0,882 & 0,922 & 0,806 & 0,938 & 0,910 & 0,364 & 0,912 & 0,945 & 0,920 & 0,670 & 0,842 \\
\hline & 3 & 0,038 & 0,029 & 0,018 & 0,060 & 0,019 & 0,018 & 0,074 & 0,607 & 0,039 & 0,027 & 0,080 & 0,330 & 0,112 \\
\hline \multirow[t]{2}{*}{ GTDH1 } & 1 & 0,037 & 0,000 & 0,000 & 0,000 & 0,000 & 0,000 & 0,016 & 0,000 & 0,000 & 0,000 & 0,018 & 0,027 & 0,008 \\
\hline & 2 & 0,963 & 1,000 & 1,000 & 1,000 & 1,000 & 1,000 & 0,984 & 1,000 & 1,000 & 1,000 & 0,982 & 0,973 & 0,992 \\
\hline G2DH1 & 1 & 1,000 & 1,000 & 1,000 & 1,000 & 1,000 & 1,000 & 1,000 & 1,000 & 1,000 & NA & 1,000 & 1,000 & 1,000 \\
\hline \multirow[t]{3}{*}{ DIA2 } & 1 & 0,815 & 0,000 & 0,889 & 1,000 & 0,891 & 1,000 & 1,000 & 0,707 & 0,980 & 1,000 & 0,759 & 0,973 & 0,835 \\
\hline & 2 & 0,185 & 1,000 & 0,111 & 0,000 & 0,109 & 0,000 & 0,000 & 0,279 & 0,020 & 0,000 & 0,196 & 0,027 & 0,161 \\
\hline & 3 & 0,000 & 0,000 & 0,000 & 0,000 & 0,000 & 0,000 & 0,000 & 0,014 & 0,000 & 0,000 & 0,045 & 0,000 & 0,005 \\
\hline \multirow[t]{3}{*}{ G6PDH1 } & 1 & 0,000 & 0,000 & 0,000 & 0,000 & 0,000 & 0,143 & 0,000 & 0,000 & 0,000 & 0,000 & 0,000 & 0,000 & 0,012 \\
\hline & 2 & 0,905 & 1,000 & 1,000 & 1,000 & 0,880 & 0,839 & 0,984 & 1,000 & 0,961 & 0,955 & 0,982 & 1,000 & 0,959 \\
\hline & 3 & 0,095 & 0,000 & 0,000 & 0,000 & 0,120 & 0,018 & 0,016 & 0,000 & 0,039 & 0,045 & 0,018 & 0,000 & 0,029 \\
\hline \multirow[t]{3}{*}{ IDH1 } & 1 & 0,000 & 0,000 & 0,009 & 0,000 & 0,000 & 0,000 & 0,000 & 0,007 & 0,000 & 0,000 & 0,000 & 0,009 & 0,002 \\
\hline & 2 & 1,000 & 1,000 & 0,991 & 0,974 & 1,000 & 1,000 & 1,000 & 0,979 & 1,000 & 1,000 & 1,000 & 0,991 & 0,995 \\
\hline & 3 & 0,000 & 0,000 & 0,000 & 0,026 & 0,000 & 0,000 & 0,000 & 0,014 & 0,000 & 0,000 & 0,000 & 0,000 & 0,003 \\
\hline \multirow[t]{4}{*}{ PGI3 } & 1 & 0,000 & 0,019 & 0,000 & 0,000 & 0,000 & 0,000 & 0,000 & 0,000 & 0,000 & 0,018 & 0,000 & 0,000 & 0,003 \\
\hline & 2 & 0,000 & 0,019 & 0,000 & 0,000 & 0,000 & 0,000 & 0,000 & 0,000 & 0,000 & 0,018 & 0,000 & 0,000 & 0,003 \\
\hline & 3 & 1,000 & 0,962 & 1,000 & 1,000 & 1,000 & 1,000 & 1,000 & 0,993 & 1,000 & 0,964 & 1,000 & 0,973 & 0,991 \\
\hline & 4 & 0,000 & 0,000 & 0,000 & 0,000 & 0,000 & 0,000 & 0,000 & 0,007 & 0,000 & 0,000 & 0,000 & 0,027 & 0,003 \\
\hline
\end{tabular}


Foram detectados, para as 12 populações, 16 alelos com frequência menor que $5 \%$ no conjunto das populações, totalizando $50 \%$ do total de alelos encontrados. Além do mais, foram detectados dois alelos exclusivos, um na população São José do Cerrito (G6PD1-1) e outro na população Campo Alegre (MDH1-2).

Analisando-se as frequências alélicas dentro das microrregiões foram detectados dois alelos exclusivos na microrregião dos Campos de Lages (MDH1-1 e MDH1-5), sendo que os dois ocorrem na população de São José do Cerrito e o segundo ocorre também na população de Capão Alto. Além destes ainda há um alelo que ocorre somente na microrregião de Mafra, sendo ele exclusivo da população de Campo Alegre (MDH1-2).

As populações de Podocarpus lambertii estudadas estão localizadas em três Bacias Hidrográficas, a Timbó/Canoinhas/Negro, Rio Canoas e a Irani/Jacutinga/Peixe que conta somente com a população Caçador. Enquanto a Bacia Timbó/Canoinhas/Negro apresentou somente um alelo exclusivo (MDH1-2) na população de Campo Alegre, foram detectados quatro alelos exclusivos da Bacia do Rio Canoas, sendo estes os alelos MDH1-5, PGM1-4, G6PD1-1 e PGI3-4.

A partir das frequências alélicas foram estimados os índices de diversidade genética e de fixação para as 12 populações de Podocarpus lambertii (Tabela 3). A porcentagem de locos polimórficos foi de $52,7 \%$, valor similar aos encontrados para Podocarpus parlatorei $(P=42 \%)$ por Quiroga e Premoli (2007) e para Podocarpus nubigena ( $P=64,9 \%$ ) por Quiroga e Premoli (2010).

Tabela 3 - Índices de diversidade genética ( $\mathrm{P}$ - Porcentagem de locos polimórficos, A - Número de alelos por loco, $\mathrm{H}_{0}$ - Heterozigosidade observada, $\mathrm{H}_{\mathrm{e}}-$ Heterozigosidade esperada, $\mathrm{f}$ - índice de fixação, AR - Número de alelos raros, AE - Número de alelos exclusivos) para 12 populações, e suas respectivas microrregiões, de Podocarpus lambertii no Estado de Santa Catarina. UFSC, 2011.

Table 3 - Genetic diversity indexes (percentage of polymorphic loci - $P$, number of allele per locus - $A$, genetic diversity - $H_{\mathrm{e}}$, observed heterozygosis - $H_{\mathrm{o}}$, fixation index $-f$, rare alleles - AR and exclusive alleles $-\mathrm{AE}$ ) for 12 populations and their respective microregions of Podocarpus lambertii in Santa Catarina state. UFSC, 2011.

\begin{tabular}{|c|c|c|c|c|c|c|c|c|c|}
\hline Microrregião & População & $\mathbf{P}$ & $\mathbf{A}$ & Ho & $\mathrm{He}$ & f & & AR & $\mathbf{A E}$ \\
\hline \multirow[t]{4}{*}{ Curitibanos } & São Cristovão1 - 453 & 63,6 & 1,82 & 0,059 & 0,097 & 0,388 & $*$ & 6 & 0 \\
\hline & Curitibanos -561 & 54,5 & 1,91 & 0,091 & 0,096 & 0,052 & & 8 & 0 \\
\hline & Curitibanos 2-409 & 54,5 & 1,82 & 0,053 & 0,071 & 0,247 & $*$ & 6 & 0 \\
\hline & Média & 57,6 & 1,85 & 0,07 & 0,088 & 0,207 & & - & - \\
\hline \multirow[t]{4}{*}{ Joaçaba } & Lebon Régis $2-619$ & 63,6 & 1,82 & 0,05 & 0,093 & 0,457 & $*$ & 6 & 0 \\
\hline & Lebon Régis $1-789$ & 54,5 & 1,55 & 0,024 & 0,059 & 0,597 & $*$ & 4 & 0 \\
\hline & Caçador - 784 & 45,5 & 1,73 & 0,039 & 0,039 & 0,015 & & 6 & 0 \\
\hline & Média & 54,5 & 1,7 & 0,038 & 0,063 & 0,399 & & - & - \\
\hline \multirow{4}{*}{ Campos de Lages } & Painel - 211 & 45,5 & 1,55 & 0,052 & 0,092 & 0,436 & $*$ & 3 & 0 \\
\hline & São José do Cerrito - 297 & 45,5 & 1,73 & 0,016 & 0,064 & 0,744 & $*$ & 6 & 1 \\
\hline & Capão Alto - 208 & 60 & 2,2 & 0,042 & 0,062 & 0,32 & $*$ & 10 & 0 \\
\hline & Média & 50,3 & 1,82 & 0,036 & 0,068 & 0,48 & & - & 2 \\
\hline \multirow[t]{5}{*}{ Canoinhas } & Mafra - 1042 & 54,5 & 1,82 & 0,045 & 0,078 & 0,431 & $*$ & 6 & 0 \\
\hline & Bela Vista do Toldo - & & & & & & & & \\
\hline & 1010 & 45,5 & 1,73 & 0,036 & 0,084 & 0,571 & $*$ & 7 & 0 \\
\hline & Campo Alegre - 1063 & 45,5 & 1,91 & 0,085 & 0,11 & 0,224 & $*$ & 7 & 1 \\
\hline & Média & 48,5 & 1,82 & 0,054 & 0,089 & 0,387 & & - & 1 \\
\hline \multirow[t]{2}{*}{ Estado } & Média & 52,7 & 1,8 & 0,049 & 0,079 & 0,374 & & - & - \\
\hline & Total & 671,3 & 2,91 & 0,049 & 0,107 & 0,539 & & 18 & \\
\hline
\end{tabular}

Em que: $* \mathrm{P}<0,05$. Valores significativamente diferentes de zero após correção de Bonferroni. 
O número médio de alelos obtido para as populações foi de 1,8. Esse valor é muito próximo ao encontrado para outras espécies do gênero Podocarpus que ocorrem na América do Sul. Quiroga e Premoli (2007) obtiveram para Podocarpus parlatorei um número médio de alelos por população igual a 1,6 enquanto que Quiroga e Premoli (2010) obtiveram para Podocarpus nubigena um valor de $A$ igual a 1,8 .

O índice de diversidade genética $\left(H_{e}\right)$ estimado para o conjunto de populações de Podocarpus lambertii foi relativamente baixo. $\mathrm{O}$ índice $H_{e}$ obtido $(0,079)$ foi inferior aos encontrados para P. parlatorei $(0,160)$ (QUIROGA; PREMOLI, 2007) e para Podocarpus nubigena (0,222) (QUIROGA; PREMOLI, 2010).

A heterozigosidade média observada foi ainda mais baixa com uma média de 0,049 . Essa diferença gerou um índice de fixação médio para as populações de 0,374 . Este índice de fixação médio obtido para as populações é alto e indica um deficit de heterozigotos grande dentro das populações da espécie. Somente duas populações apresentaram índices de fixação baixos e não significativamente diferentes de zero, a população Curitibanos-1 e a população Caçador. Este resultado indica que as duas populações apresentam as proporções genotípicas dentro do esperado em Equilíbrio de Hardy-Weinberg.

Analisando os índices de estrutura genética de populações propostos por Weir e Cockerham (1984) verifica-se uma grande divergência genética entre as populações de Podocarpus lambertii (Tabela 4) com $F_{S T}$ estimado de 0,303 . O valor do $G_{S T}$ ' de Hedrick, que leva em consideração no cálculo da divergência genética a composição alélica das populações (diferentes tipos alélicos), não diferiu muito e foi estimado em 0,355. Estes valores são muito superiores aos índices $F_{S T}$ obtidos por Quiroga e Premoli $(2007 ; 2010)$ para Podocarpus parlatorei $(0,104)$ e para Podocarpus nubigena $(0,181)$.

\section{Tabela 4 - Estatísticas F de Wright, obtidos pelo método de variância (WEIR; COCKERHAM, 1984) para 12 populações de Podocarpus lambertii, separadas por microrregiões e bacias hidrográficas. UFSC, 2011.}

Table 4 - Wright's F-statistics, obtained by the variance method (WEIR; COCKERHAM, 1984) for 12 populations of Podocarpus lambertii, subdivided in microrregions and hidrographic basins. UFSC, 2011.

\begin{tabular}{lccc|c|c}
\hline \multicolumn{1}{c|}{ Microrregião } & FIT (IC 95\%) & FST (IC 95\%) & FIS (IC 95\%) & GST \\
\hline Curitibanos & $0,544(0,287$ a 0,707$)$ & $0,424(0,038$ a 0,617$)$ & $0,207(0,059$ a 0,388$)$ & 0,506 \\
Joaçaba & $0,461(0,270$ a 0,694$)$ & $0,103(0,035$ a 0,131$)$ & $0,399(0,211$ a 0,671$)$ & 0,117 \\
Campos de Lages & $0,500(0,318$ a 0,645$)$ & $0,038(0,018$ a 0,057$)$ & $0,480(0,296$ a 0,630$)$ & 0,044 \\
Canoinhas & $0,646(0,198$ a 0,949$)$ & $0,421(0,028$ a 0,774$)$ & $0,387(0,094$ a 0,835$)$ & 0,505 \\
Bacias & FIT (IC 95\%) & FST (IC $95 \%)$ & FIS (IC $95 \%)$ & GST \\
Timbó & $0,652(0,278$ a 0,924$)$ & $0,396(0,079$ a 0,727$)$ & $0,424(0,145$ a 0,783$)$ & 0,475 \\
Canoas & $0,527(0,397$ a 0,655$)$ & $0,280(0,033$ a 0,455$)$ & $0,343(0,214$ a 0,544$)$ & 0,327 \\
Total & $0,550(0,370$ a 0,713$)$ & $0,303(0,073$ a 0,430$)$ & $0,355(0,208$ a 0,581$)$ & 0,355 \\
\hline
\end{tabular}

A forte estruturação encontrada para o conjunto de populações $\left(G_{S T}{ }^{\prime}=0,355\right)$ pode indicar que o fluxo gênico histórico entre as mesmas foi muito baixo. No entanto, as populações presentes dentro da microrregião dos Campos de Lages apresentaram uma estruturação menor $\left(G_{S T}{ }^{\prime}=0,044\right)$. Esse índice indica que para essas populações a estruturação é fraca, sugerindo que o fluxo gênico pode estar atuando fortemente contra os efeitos da deriva genética nestas populações. Este pode ser um indício de que a fragmentação florestal nessa microrregião não afetou drasticamente o fluxo gênico entre essas populações ou, ainda, que não houve uma redução dos tamanhos populacionais tão drástica. No entanto, deve-se considerar que o processo exploratório nesta região teve início mais tarde do que nas demais regiões 
do Estado. Com isso, há a possibilidade de que ainda não houve tempo suficiente para que os efeitos do processo exploratório pudessem ser captados por esta metodologia. Uma análise hierarquizada AMOVA foi realizada e indicou que, apesar das diferenças entre as populações dentro das bacias já discutidas, não há diferenças significativas entre as bacias.

Para as 12 populações analisadas de Podocarpus lambertii não foi encontrada correlação entre o Log das distâncias geográficas e o $F_{S T} /\left(1-F_{S T}\right)\left(\mathrm{R}^{2}=0,028\right)$ (Figura 2). Esse resultado indica que o isolamento por distância não explica o padrão de estruturação genética encontrado para Podocarpus lambertii. Outros fatores como a conectividade, rotas migratórias, efeito fundador e deriva genética devem ter influenciado o padrão de estruturação genética entre as populações de Podocarpus lambertii.

$\mathrm{O} F_{I S}$ (Tabela 5) estimado para o conjunto das populações de 0,355 indica um grande deficit de heterozigotos no conjunto das populações. Quiroga e Premoli (2010) encontraram para 14 populações Podocarpus nubigena, uma espécie também dioica, um índice $F_{I S}$ de 0,258 . Contudo, para 18 populações de Podocarpus parlatorei o índice de fixação obtido foi de $-0,104$, indicando que essa última espécie apresenta, ao contrário do Podocarpus lambertii, um excesso de heterozigotos. $\mathrm{O}$ valor do índice de fixação estimado para Podocarpus lambertii é muito alto, principalmente para uma espécie dioica, incapaz de realizar autofecundação. Portanto, esse alto índice de fixação deve ser decorrente principalmente de cruzamento entre aparentados. É possível que, parte desse índice de fixação seja decorrente da existência de estrutura genética interna e consequentemente do efeito Wahlund. Neste caso seria importante que trabalhos que caracterizem a estrutura genética espacial interna de populações da espécie sejam desenvolvidos para testar esta hipótese. A redução do tamanho dos remanescentes florestais, assim como o corte seletivo de indivíduos da espécie, que resultam em uma redução do número de indivíduos reprodutivos e consequentemente uma redução de oportunidades de cruzamentos, também podem ser fatores que contribuem para estes elevados índices encontrados, visto que muitos dos indivíduos coletados devem ter sido gerados após a intensificação do processo exploratório, iniciada nessas regiões no início do século passado.

Figura 2 - Correlação de Mantel entre o logaritmo natural da distância geográfica e o $\mathrm{F}_{\mathrm{ST}} /\left(1-\mathrm{F}_{\mathrm{ST}}\right)$ par a par para 12 populações de Podocarpus lambertii no Estado de Santa Catarina. UFSC, 2011.

Figure 2 - Mantel correlation between the natural logarithm of geographic distance and pairwise $F_{S T} /\left(1-F_{S T}\right)$ for 12 populations of Podocarpus lambertii in Santa Catarina state. UFSC, 2011.

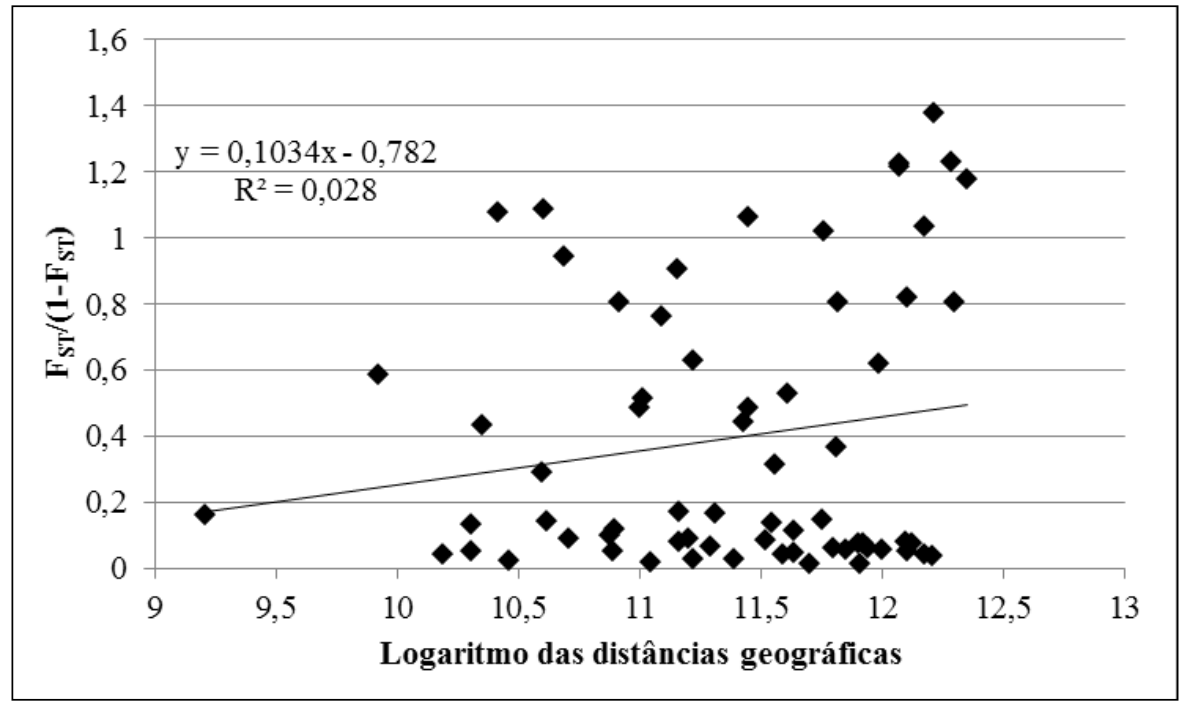


Figura 3 - Dendrograma de distâncias genéticas de Nei (1972) e proporção de réplicas similares por reamostragem bootstrap (10.000 repetições) em cada nó para 12 populações de Podocarpus lambertii no Estado de Santa Catarina. UFSC, 2011.

Figure 3 -Pairwise genetic distances Nei (1972) and proportion of similar bootstrap replicates (10.000 resampling) for 12 Podocarpus lambertii populations in Santa Catarina state. UFSC, 2011.

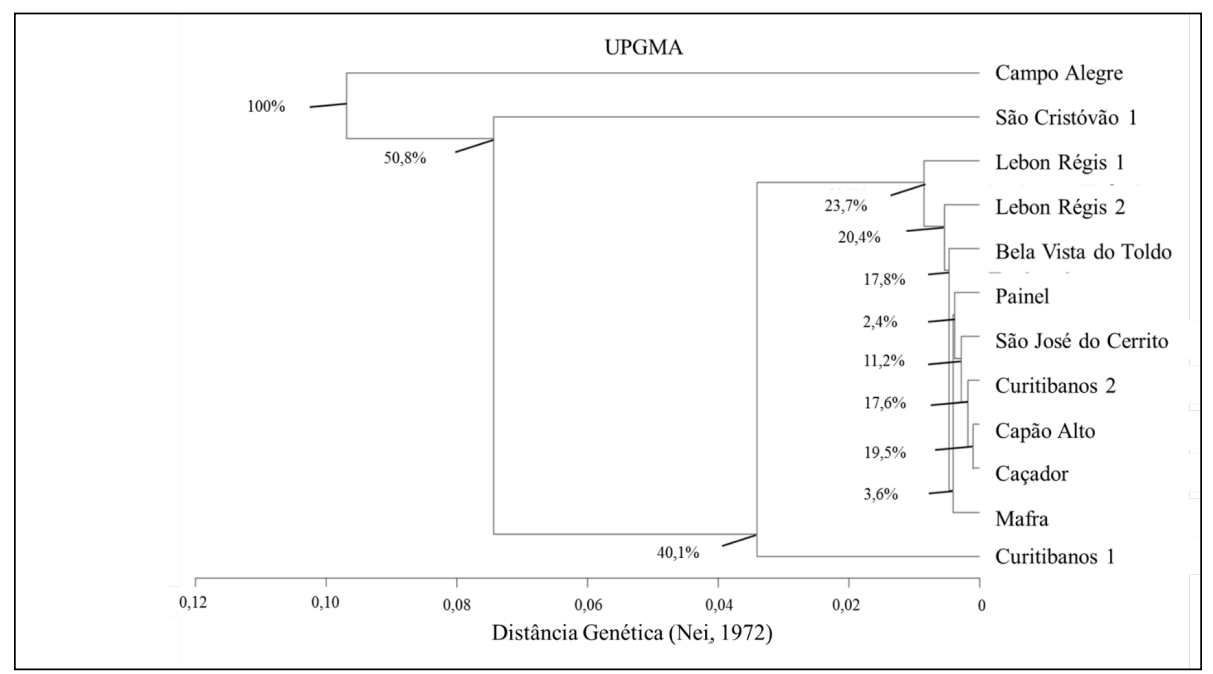

Tendo por base a estimativa de distância genética entre populações proposta por Nei (1972) e através do método UPGMA foi gerado um dendrograma de distâncias genéticas para as 12 populações de Podocarpus lambertii analisadas (Figura 3).

É possível observar um grupo bastante semelhante geneticamente, apesar dos baixos valores de proporção de réplicas similares (bootstrap), formado pelas populações Capão Alto, Caçador, Curitibanos-2, São José do Cerrito, Painel, Mafra, Bela Vista do Toldo e Lebón Régis 1 e 2. Entretanto, as outras populações (Campo Alegre, São Cristóvão-1 e Curitibanos-1) apresentam distâncias maiores. Deste modo, estas últimas populações têm grande importância no que se refere à conservação da espécie. Programas de conservação devem levar em conta a importância destas três populações mais distantes geneticamente, juntamente com algumas do grupo mais similar.

As populações localizadas na microrregião de Campos de Lages, principalmente a população São José do Cerrito e Capão Alto apresentam também um papel importante na conservação da diversidade genética da espécie, pois, apresentam dois alelos exclusivos da região. Do mesmo modo, outra população importante para a conservação in situ é a população Campo Alegre, pois além de apresentar um alelo exclusivo, é a população com o maior índice de diversidade genética.

As populações Curitibanos e Caçador são muito importantes devido ao fato de que são as únicas que apresentaram índices de fixação baixos e não diferentes de zero, indicando que estas populações aderem ao esperado em Equilíbrio de Hardy-Weinberg. Desse modo, estas populações apresentam grande potencial evolutivo, pois as chances de estas sofrerem com os efeitos da endogamia e deriva genética, que afetam negativamente a viabilidade de populações pequenas (FRANKHAM, 2005), são menores do que nas outras populações que apresentam índices de fixação significativos.

Contudo, as outras populações, apesar de não serem prioritárias, também podem apresentar um importante papel na garantia do potencial adaptativo da espécie no longo prazo, visto que grande parte da variabilidade genética é decorrente da divergência genética entre as populações. A manutenção desta variação é de suma importância para a espécie visto que os valores de diversidade genética da mesma são relativamente baixos.

É importante ressaltar que neste trabalho foram utilizados indivíduos adultos e como a espécie trabalhada é longeva, os resultados obtidos são de acontecimentos históricos. Desse modo, os níveis de diversidade e a estruturação das populações são reflexos de eventos reprodutivos ocorridos, em alguns casos antes e em outros casos poucas décadas após, o início do forte processo exploratório no Estado. 
Levando isso em consideração e o atual quadro de conservação dos remanescentes florestais da Floresta Ombrófila Mista no estado, as gerações que estão sendo geradas atualmente tendem a apresentar um estado mais crítico do que os indivíduos adultos analisados.

\section{Conclusões}

Como foi detectado neste trabalho as populações de Podocarpus lambertii apresentam uma forte estruturação genética, o que indica uma deficiência no fluxo gênico histórico entre as populações. Além do mais, metade dos alelos detectados ocorre em baixa frequência, portanto, estão muito susceptíveis aos efeitos da deriva genética. Por conseguinte, sugere-se que sejam implementadas ações para aumentar a conectividade entre as populações de Podocarpus lambertii, visando à conservação a médio e longo prazo das populações da espécie. Visto que o fluxo gênico contrapõe os efeitos da deriva genética, uma maior conectividade poderá garantir a persistência dos alelos raros, que são mais susceptíveis a flutuações aleatórias que possam ser decorrentes do tamanho reduzido dos remanescentes florestais.

Ações envolvendo a recuperação de áreas degradadas no entorno dos remanescentes florestais podem aumentar o tamanho populacional das espécies florestais assim como o aumento da conectividade entre os fragmentos podem auxiliar na conservação in situ da espécie. A introdução de migrantes, vindo de áreas próximas para evitar problemas de depressão por cruzamento, também pode auxiliar nesse sentido.

Os resultados obtidos também apontam a necessidade de uma política estadual para a produção de mudas de espécies florestais nativas, visando aos plantios de restauração e comerciais. A alta estruturação genética encontrada indica que as coletas de sementes para produção de mudas visando à recuperação de áreas degradadas, devem ser feitas sempre em áreas próximas ao local em que serão implantadas as mesmas, caso contrário, os riscos de depressão por exogamia podem ser grandes.

Há também uma grande variação nos níveis de diversidade genética e nos índices de fixação nas populações. Portanto, a indicação de áreas propícias para coleta de sementes poderia maximizar o potencial das novas populações a serem fundadas. No entanto, esse tipo de trabalho deve ser continuado, com capacitação de pessoal, tanto de viveiristas quanto de agentes do Estado e criação de uma regulamentação para a produção de mudas de espécies florestais nativas.

\section{Agradecimentos}

Os autores deste trabalho agradecem ao pessoal do Núcleo de Pesquisas de Pesquisas em Florestas Tropicais da Universidade Federal de Santa Catarina pela ajuda no laboratório e na coleta de material em campo. Também aos órgãos financiadores: Coordenação de Aperfeiçoamento de Pessoal de Nível Superior (Capes), a Fundação de Amparo à Pesquisa e Inovação do Estado de Santa Catarina (FAPESC) e o Conselho Nacional de Desenvolvimento Científico e Tecnológico (CNPq).

\section{Referências}

ALFENAS, A. C. (Ed.). Eletroforese de isoenzimas e proteínas afins: fundamentos e aplicações em plantas e microorganismos. Viçosa, MG: UFV, 1998. 574 p.

BARRET, S. C. H.; KOHN, J. R. Genetic and evolutionary consequences of small population size in plants: implications for conservation. In: FALK, D. A.; HOLSINGER, K. E. (Ed.). Genetics and conservation of rare plants. Oxford: Oxford University Press, 1991. P. 3-30.

BERG, E. E.; HAMRICK, J. L. Quantification of genetic diversity at allozyme loci. Canadian Journal of Forest Research, Ottawa, v. 27, p. 415-424, 1997.

CARVALHO, P. E. R. Espécies florestais brasileiras: recomendações silviculturais, potencialidades e uso da madeira. Colombo: EMBRAPA; CNPF, 1994.

CLARKE, G. M.; YOUNG, A. G. Introduction: genetics, demography and the conservation of fragmented populations. In: YOUNG, A. G.; CLARKE, G. M. (Ed.). Genetics, demography and viability of 
fragmented populations. Cambridge: Cambridge University Press, 2000. p. 1-7.

COLE, C. T. Genetic variation in rare and common plants. Annual Review of Ecology, Evolution, and Systematics, Palo Alto, v. 34 p. 213-237, 2003.

DUDASH, M. R.; FENSTER, C. B. Inbreeding and outbreeding depression in fragmented populations. In: YOUNG, A. G.; CLARKE, G. M. (Ed.). Genetics, demography and viability of fragmented populations. Cambridge: Cambridge University Press, 2000. p. 35-43.

ELAM, R.; ELLSTRAND, N. C. Population genetic consequences of small population size: implications for plant conservation. Annual Review of Ecology and Systematics, Palo Alto, v. 24, p. 217-242, 1993.

FRANKHAM, R. Genetics and extinction. Biological Conservation, Essex, v. 126, p. 131-140, 2005.

FUNDAÇÃO SOS MATAATLÂNTICA; INSTITUTO NACIONAL DE PESQUISAS ESPACIAIS (Brasil). Atlas dos remanescentes florestais da Mata Atlântica no período 2008-2010. São Paulo: [s.n.], 2011.

GOUDET, J. Fstat (Version 2.9.3.2.): a computer program to calculate F-statistics. Journal of Heredity, Newport, v. 86, p. 485-486, 2002.

HAMRICK, J. L.; GODT, M. J. W. Allozyme diversity in plant species. In: BROWN, A. H. D. et al. (Ed.). Plant population genetics, breeding and genetic resources. Sunderland: Sinauer, 1989. p. 43-63.

HEDRICK, F. A standardized genetic differentiation measured. Evolution, Medford, v. 59, p. 1633-1638, 2005.

LEWIS, P. O.; ZAYKIN, D. Genetic data analysis: computer program for the analysis of allelic data. Version 1.0 (d16c). 2001. Disponível em: http://lewis.eeb.uconn.edu/ lewishome/ software.html.

LONGHI, R. A. Livro das árvores: árvores e arvoretas do Sul. Porto Alegre: L\&PM, 1995. 176 p.

LONGHI, S. J. et al. Fatores ecológicos determinantes na ocorrência de Araucaria angustifolia e Podocarpus lambertii, na Floresta Ombrófila Mista da FLONA de São Francisco de Paula, RS, Brasil. Ciência Rural, Santa Maria, v. 40, n. 1, p. 57-63, 2010.

MYERS, N. et al. Biodiversity hotspots for conservation priorities. Nature, London, v. 403, p. 853-858, 2000.

NEI, M. Genetic distance between populations. American Naturalist, Chicago. v. 106, p. 283-29, 1972.

QUIROGA, M. P.; PREMOLI, A. Genetic patterns in Podocarpus parlatorei reveal the long-term persistence of cold tolerant elements in the southern Yungas. Journal of Biogeography, Medford, v. 34, p. 447-455, 2007.

QUIROGA, M. P.; PREMOLI, A. Genetic structure of Podocarpus nubigena (Podocarpaceae) provides evidence of Quaternary and ancient historical events. Palaeogeography, Palaeoclimatology, Palaeoecology, Amsterdam, v. 285, p. 186-193, 2010.

RIBEIRO, S. B. et al. Diversidade e classificação da comunidade arbórea da Floresta Ombrófila Mista da flona de São Francisco de Paula, RS. Ciência Florestal, Santa Maria, v. 17, n. 2, p. 101-108, 2007

ROHLF, F. J. NTSYS-pc: numerical taxonomy and multivariate analysis system. New York: Exeter Publisher, 1989.

THRALL, P. H.; BURDON, J. J.; MURRAY, B. R. The metapopulation paradigm: a fragmented view of conservation biology. In: YOUNG, A. G.; CLARKE, G. M. (Ed.). Genetics, demography and viability of fragmented populations. Cambridge: Cambridge University Press, 2000. p. 75-95.

WEIR, B. S.; COCKERHAM, C. C. Estimating F-statistics for the analysis of population structure. Evolution, Medford, v. 38, p. 1358-1370, 1984.

YOUNG, A. G.; BOYLE, T.; BROWN, T. The population genetic consequences of habitat fragmentation for plants. Tree, Maryland Heights, v. 11, n. 10, p. 413-418, 1996.

YOUNG, A. G.; BROWN, A. H. D. Paternal bottlenecks in fragmented populations of the grassland daisy Rutidosis leptorrhynchoides. Genetics Research Cambridge, Cambridge, v. 73, p. 111-117, 1999. 